\title{
Inhibition of microRNA-492 attenuates cell proliferation and invasion in retinoblastoma via directly targeting LATS2
}

\author{
ZHIQUN SUN, AIMEI ZHANG and LIMING ZHANG \\ Department of Pediatrics, Weifang People's Hospital, Weifang, Shandong 261000, P.R. China
}

Received April 23, 2018; Accepted November 22, 2018

DOI: $10.3892 / \mathrm{mmr} .2018 .9784$

\begin{abstract}
Numerous studies have demonstrated that microRNAs (miRNAs) are upregulated or downregulated in retinoblastoma ( $\mathrm{RB})$, and that this phenomenon is associated with the modulation of various malignant behaviours during RB occurrence and development. Therefore, the mechanisms that associate deregulated miRNAs with RB initiation and progression must be understood to identify effective therapeutic techniques for patients with $\mathrm{RB}$. In the present study, miR-492 expression was upregulated in RB tissues and cell lines. The effects of miR-492 inhibition on the proliferation and invasion of RB cells were examined using Cell Counting kit- 8 and invasion assays. The results revealed that miR-492 downregulation significantly decreased the proliferation and invasion of RB cells. Bioinformatics analysis predicted that large tumour-suppressor kinase 2 (LATS2) was a putative target of miR-492. Luciferase reporter assay, reverse transcription-quantitative polymerase chain reaction and western blot analysis demonstrated that LATS2 was a direct target gene of miR-492 in RB cells. In addition, LATS2 expression was downregulated in RB tissues, and its downregulation was inversely correlated with miR-492 level. Furthermore, LATS2-knockdown abrogated the effects of miR-492 downregulation in RB cells. In conclusion, miR-492 inhibition may impede the malignant behaviour of RB by directly targeting LATS2. Therefore, targeting this miRNA may be an effective therapeutic method for treating patients with RB.
\end{abstract}

\section{Introduction}

Retinoblastoma (RB) is the most common intraocular malignancy in infants and young children (1). RB accounts for $2-4 \%$ of all paediatric cancer types, and the high-risk age

Correspondence to: Professor Aimei Zhang, Department of Pediatrics, Weifang People's Hospital, 151 Guangwen Road, Weifang, Shandong 261000, P.R. China

E-mail: zhang_aimei151@126.com

Key words: retinoblastoma, microRNA-492, proliferation, invasion, large tumour-suppressor kinase 2 of developing RB is 5 years old $(2,3)$. Defective RB tumour suppressor gene 1 (Rb1) is the main factor involved in the initiation and progression of RB (4). However, the detailed mechanisms underlying the pathogenesis and progression of $\mathrm{RB}$ remain largely unknown. At present, the primary therapeutic techniques for patients with RB include ophthalmectomy, laser photocoagulation, cryotherapy, hyperthermia and chemoradiotherapy (5). The majority of patients with RB are not diagnosed at an early stage, and their delayed diagnosis is associated with the high incidence of mortality in this patient group (6). The overall survival rate of patients with $\mathrm{RB}$ is extremely poor, despite improvements in the applied therapeutic methods (7). Therefore, the molecular mechanisms by which RB is initiated and the factors that drive development must be elucidated to develop effective diagnostic markers and intervention approaches for managing patients with RB.

microRNAs (miRNAs) are a family of non-coding and evolutionarily conserved RNAs. miRNAs contain 18-24 nucleotides, and are associated with the regulation of genes expression (8). miRNAs can inhibit gene expression by binding miRNA 'seed' regions to complementary sequences of 3'-untranslational regions (3'-UTRs) of their target genes, thereby causing mRNA degradation and/or mRNA translation suppression (9). Over the past decade, studies have revealed that miRNAs are altered in nearly all human cancer types, and that their alteration contributes toward the initiation and progression of cancer (10-12). An increasing volume of evidence has recently indicated that numerous miRNAs, including miR-29a (13), miR-138 (14), miR-498 (15) and miR-655 (16), are aberrantly expressed in RB. miRNA dysregulation participates in the regulation of various biological behaviours, including cellular proliferation, cycle, apoptosis and invasion, as well as metastasis and angiogenesis (17-19). Therefore, miRNAs may serve as potential diagnostic biomarkers and therapeutic targets for patients with RB.

miR-492 is reportedly downregulated in cervical cancer (20), osteosarcoma (21) and clear cell renal cell carcinoma (22). By contrast, miR-492 is upregulated in hepatoblastoma, hepatic cancer (23) and breast cancer (24). However, the expression pattern and detailed roles of miR-492 in RB and its regulation mechanisms remain largely unclear. Therefore, the present study aimed to detect miR-492 expression in RB, to investigate the detailed roles of miR-492 in RB progression and to identify the molecular mechanism underlying the action of miR-492 in RB. 


\section{Patients and methods}

Patients and tissue collection. RB tissues were obtained from 27 patients with RB (16 males and 11 females; age range, 13-46 years; mean age, 24 years) who had undergone surgery at Weifang People's Hospital between February 2015 and March 2017. A total of 10 normal retinal tissues were obtained from patients (6 males and 4 females; age range, 24-58 years; mean age, 31 years) who had had a globe rupture and had undergone an ophthalmectomy. All tissues were quickly stored in liquid nitrogen until further use. None of the patients had been treated with radiotherapy, chemotherapy or any other treatments prior to surgical resection. The present study was approved by the Ethics Committee of Weifang People's Hospital and all subjects had provided written informed consent.

Cell lines. Three human RB cell lines (Weri-RB1, SO-RB50 and Y79) and a normal retinal pigmented epithelial cell line (ARPE-19) were acquired from American Type Culture Collection (Manassas, VA, USA). Cells were cultured in Dulbecco's modified Eagle's medium (DMEM) with $10 \%$ fetal bovine serum (FBS), $100 \mathrm{U} / \mathrm{ml}$ penicillin, and $100 \mathrm{mg} / \mathrm{ml}$ streptomycin (all Gibco; Thermo Fisher Scientific, Inc., Waltham, MA, USA), and grown at $37^{\circ} \mathrm{C}$ under conditions of $100 \%$ humidity, $95 \%$ air and $5 \% \mathrm{CO}_{2}$.

miRNA inhibitor and short interfering RNA (siRNA) transfection. miR-492 inhibitor and negative control miRNA inhibitor (NC inhibitor) were designed and produced by GenePharma Co., Ltd. (Shanghai, China). The sequences were as follows: miR-492 inhibitor, 5'AAGAAUCUUGUC CCGCAGGUCCU3'; NC inhibitor, 5'CAGUACUUUUGU GUAGUACAA3'; LATS2 siRNA targeting the expression of LATS2 (LATS2-siRNA) and negative control siRNA (NC-siRNA) were purchased from Guangzhou RiboBio Co., Ltd. (Guangzhou, China). The LATS2-siRNA sequence was 5'GTTCGGACCTTATCAGAAA3' and the NC-siRNA sequence was 5'UUCUCCGAACGUGUCACGUTT3'. Cells were inoculated into 6-well culture plates one day prior to transfection. miRNA inhibitor or siRNA was transfected into cells at a concentration of $100 \mathrm{nM}$ using Lipofectamine 2000 (Invitrogen; Thermo Fisher Scientific, Inc.), according to the manufacturer's protocols. Reverse transcription-quantitative polymerase chain reaction (RT-qPCR) and western blot analysis was performed at 48 and $72 \mathrm{~h}$ post-transfection. Cell Counting kit-8 (CCK-8) and invasion assays were carried out after 24 and $48 \mathrm{~h}$ culture, respectively.

$R N A$ isolation and $R T-q P C R$. Total RNA was isolated from cells and tissue specimens using TRIzol reagent (Invitrogen; Thermo Fisher Scientific, Inc.). The concentration and purity of total RNA was evaluated using a Nanodrop 2000 (Thermo Fisher Scientific, Inc.). For quantification of miR-492 expression, complementary DNA (cDNA) was synthesized by reverse transcription using the TaqMan ${ }^{\circledR}$ MicroRNA Reverse Transcription kit (Applied Biosystems; Thermo Fisher Scientific, Inc.). The temperature protocol for reverse transcription was as follows: $16^{\circ} \mathrm{C}$ for $30 \mathrm{~min}, 42^{\circ} \mathrm{C}$ for $30 \mathrm{~min}$ and $85^{\circ} \mathrm{C}$ for $5 \mathrm{~min}$. The TaqMan MicroRNA assay kit (Applied Biosystems; Thermo Fisher Scientific, Inc.) was utilized to analyze miR-492 expression, using U6 snRNA as an internal reference. The cycling conditions were as follows: $50^{\circ} \mathrm{C}$ for $2 \mathrm{~min}, 95^{\circ} \mathrm{C}$ for $10 \mathrm{~min} ; 40$ cycles of denaturation at $95^{\circ} \mathrm{C}$ for $15 \mathrm{sec}$; and annealing/extension at $60^{\circ} \mathrm{C}$ for $60 \mathrm{sec}$.

For the detection of LATS2 mRNA expression, total RNA was converted into cDNA using the PrimeScript ${ }^{\mathbb{Q}} \mathrm{RT}$ reagent kit (Takara Bio, Inc., Otsu, Japan). The temperature protocol for reverse transcription was as follows: $37^{\circ} \mathrm{C}$ for $15 \mathrm{~min}$ and $85^{\circ} \mathrm{C}$ for 5 second. The expression of LATS2 mRNA was determined by the SYBR Premix ExTaq kit (Takara Bio, Inc., Otsu, Japan), using GAPDH as an internal control. The cycling conditions were as follows: $5 \mathrm{~min}$ at $95^{\circ} \mathrm{C}$, followed by 40 cycles of $95^{\circ} \mathrm{C}$ for $30 \mathrm{sec}$ and $65^{\circ} \mathrm{C}$ for $45 \mathrm{sec}$. Relative gene expression was calculated using the $2^{-\Delta \Delta \mathrm{Cq}}$ method (25). The primers were designed as follows: miR-492 forward, 5'-CTCA ACTGGTGTCGTGGAGTCGGCAATTCAGTTGAGAAGA ATCT-3' and reverse, 5'-ACACTCCAGCTGGGAGGACCT GCGGACAAG-3'; U6 forward, 5'-CTCGCTTCGGCAGCA CATATACT-3' and reverse, 5'-ACGCTTCACGAATTTGCG TGTC-3'; LATS2 forward, 5'-CCCGAGGAATGAGCAGAT TG-3' and reverse, 5'-GCTGGTGGTAGGACGCAAAC-3'; and GAPDH forward, 5'-AGGGCTGCTTTTAACTCTGGT-3' and reverse, 5'-CCCCACTTGATTTTGGAGGGA-3'.

CCK-8 assay. The proliferation ability of RB cells was determined using the CCK-8 assay. In brief, transfected cells were collected at $24 \mathrm{~h}$ post-transfection, prepared into signal cell suspensions and plated into 96-well culture plates at a density of 3,000 cells/well. Cells were then incubated at $37^{\circ} \mathrm{C}$ under conditions of $95 \%$ air and $5 \% \mathrm{CO}_{2}$ for different time periods. At each time point, $10 \mu \mathrm{l} \mathrm{CCK-8} \mathrm{(Dojindo} \mathrm{Molecular}$ Technologies, Inc., Kumamoto, Japan) was added into each well. After an additional $2 \mathrm{~h}$ incubation, the optical density (OD) value of each well was detected at a wavelength of $450 \mathrm{~nm}$ on an ELISA microplate reader (Bio-Rad Laboratories, Inc., Hercules, CA, USA).

Invasion assay. Following $48 \mathrm{~h}$ incubation, transfected cells were digested and then suspended into FBS-free DMEM. A total of $1 \times 10^{5}$ cells were added into the upper compartments of Matrigel-coated Transwell chambers (both BD Biosciences, Franklin Lakes, NJ, USA). The lower compartment of the Transwell chamber was covered with $600 \mu \mathrm{l}$ DMEM containing $20 \%$ FBS to serve as a chemoattractant. After $24 \mathrm{~h}$ incubation at $37^{\circ} \mathrm{C}$ in a $5 \% \mathrm{CO}_{2}$ atmosphere, a cotton swab was utilized to wipe out the non-invaded cells. The cells that had invaded through the polycarbonate membrane, were fixed with $100 \%$ methanol at room temperature for $20 \mathrm{~min}$ and stained with $0.1 \%$ crystal violet at room temperature for $20 \mathrm{~min}$. After being washed thrice, the number of invaded cells was counted in five randomly selected visual fields under an inverted microscope (magnification, x200; Olympus Corporation, Tokyo, Japan).

Bioinformatics prediction and luciferase reporter assay. TargetScan (Release 7.2; http://www.targetscan.org/vert_72/), miRDB (www.mirdb.org) and microRNA.org (www. microrna.org) were employed to screen the putative target. LATS2 was identified as a major target of miR-492. To experimentally verify this prediction, the 3'-UTR of LATS2 segments containing the wild-type (Wt) or mutant (Mut) 

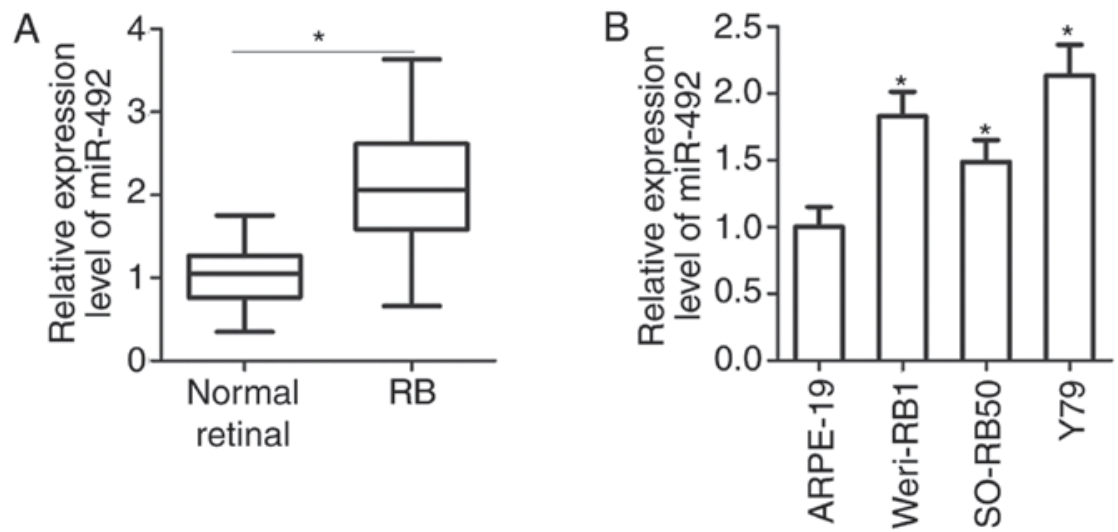

Figure 1. miR-492 expression is upregulated in RB tissues and cell lines. (A) miR-492 expression was determined in 27 RB tissues and 10 normal retinal tissues by RT-qPCR. "P<0.05 vs. normal retinal tissues. (B) RT-qPCR was utilized to detect miR-492 expression in three RB cell lines (Weri-RB1, SO-RB50 and Y79) and a normal retinal pigmented epithelial cell line (ARPE-19). $\mathrm{P}<0.05$ vs. ARPE-19.

miR-492 binding sequences were amplified by Shanghai GenePharma Co., Ltd., inserted into the pmirGLO luciferase reporter plasmid (Promega Corporation, Madison, WI, USA), and named as pmirGLO-LATS2-Wt-3'-UTR and pmirGLO-LATS2-Mut-3'-UTR, respectively. Co-transfection with pmirGLO-LATS2-Wt-3'-UTR or pmirGLO-LATS2-Mut-3'-UTR and miR-492 inhibitor or $\mathrm{NC}$ inhibitor into cells was performed using Lipofectamine 2000 , according to the manufacturer's protocols. After $48 \mathrm{~h}$ of incubation, transfected cells were collected and subjected to the detection of luciferase activities using a Dual-Luciferase Reporter assay system (Promega Corporation). Firefly luciferase activity was used for normalization.

Western blot analysis. Cells and tissues were lysed using radioimmunoprecipitation assay lysis buffer (Nanjing KeyGen Biotech Co., Ltd., Nanjing, China). Total protein concentration was detected using a BCA Protein assay kit (Nanjing KeyGen Biotech Co., Ltd.), according to the manufacturer's protocol. Equal amounts of protein $(30 \mu \mathrm{g})$ were loaded per lane, separated by $10 \%$ SDS-PAGE and transferred onto polyvinylidene fluoride membranes (EMD Millipore, Billerica, MA, USA). Subsequently, the membranes were blocked with 5\% dried skimmed milk in TBS with $0.05 \%$ Tween-20 at room temperature for $2 \mathrm{~h}$, followed by incubation with primary antibodies against LATS2 (cat. no. ab135794; dilution, 1:1,000 or GADPH (cat. no. ab186930; dilution, 1:1,000; both Abcam, Cambridge, UK). The membranes were washed three times with TBST and incubated with horseradish peroxidase-conjugated secondary antibodies (cat. no. ab6721; dilution, 1:5,000; Abcam). The protein signals were visualized using an enhanced chemiluminescence-plus reagent (GE Healthcare, Chicago, IL, USA) and quantified by densitometric analysis of protein signals using ImageJ version 1.49 (National Institutes of Health, Bethesda, MD, USA).

Statistical analysis. Data are expressed as the mean \pm standard deviation from at least three separate experiments. Differences between groups were tested using Student's t-test or one-way analysis of variance, followed by the Student-Newman-Keuls post hoc test. The correlation between miR-492 and LATS2
mRNA expression levels was assessed using Spearman's correlation analysis. All statistical analyses were conducted using SPSS 17.0 (SPSS, Inc., Chicago, IL, USA). P<0.05 was considered to indicate a statistically significant difference.

\section{Results}

High expression of miR-492 in RB tissues and cell lines. To understand the expression pattern of miR-492 in RB, its expression was detected in $27 \mathrm{RB}$ tissues and 10 normal retinal tissues. RT-qPCR analysis revealed that miR-492 was significantly upregulated in RB tissues compared with that in the 10 normal retinal tissues (Fig. $1 \mathrm{~A} ; \mathrm{P}<0.05$ ). In addition, miR-492 expression was detected in three $\mathrm{RB}$ cell lines (Weri-RB1, SO-RB50 and Y79) and one normal retinal pigmented epithelial cell line (ARPE-19). The results indicated that the expression level of miR-492 was higher in the RB cell lines than in ARPE-19 cell line (Fig. 1B; P<0.05). These results suggested that the high miR-492 expression may be associated with the malignant behaviour of RB.

miR-492 downregulation inhibits cell proliferation and invasion in $R B$. To elucidate the possible biological roles of miR-492 in RB, miR-492 or NC inhibitor was transfected into Weri-RB1 and Y79 cells, in which expression of miR-492 was relatively high. The transfection efficiency was determined by RT-qPCR. miR-492 was significantly downregulated in the Weri-RB1 and Y79 cells transfected with the miR-492 inhibitor (Fig. 2A; $\mathrm{P}<0.05)$. Next, the effect of miR-492 inhibition on the proliferation of RB cells was detected using a CCK-8 assay. As expected, miR-492 downregulation reduced the proliferative ability of Weri-RB1 and Y79 cells, compared with that in the cells transfected with $\mathrm{NC}$ inhibitor (Fig. $2 \mathrm{~B}$; $\mathrm{P}<0.05$ ). Invasion assay revealed that the invasion of miR-492 inhibitor-transfected Weri-RB1 and Y79 cells was significantly lower than that in the $\mathrm{NC}$ inhibitor group (Fig. $2 \mathrm{C} ; \mathrm{P}<0.05$ ). These results suggested that miR-492 serves oncogenic roles in the development of RB.

LATS2 is a direct target gene of miR-492 in RB cells. To investigate the molecular mechanism of miR-492 in the development of $\mathrm{RB}$, putative targets of miR-492 were screened by bioinformatics analysis. LATS2 was predicted to be a candidate of miR-492 

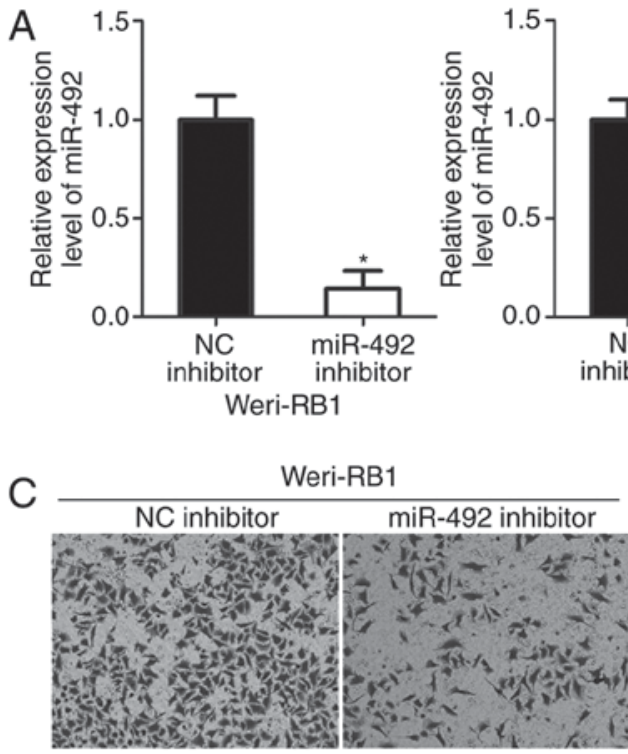
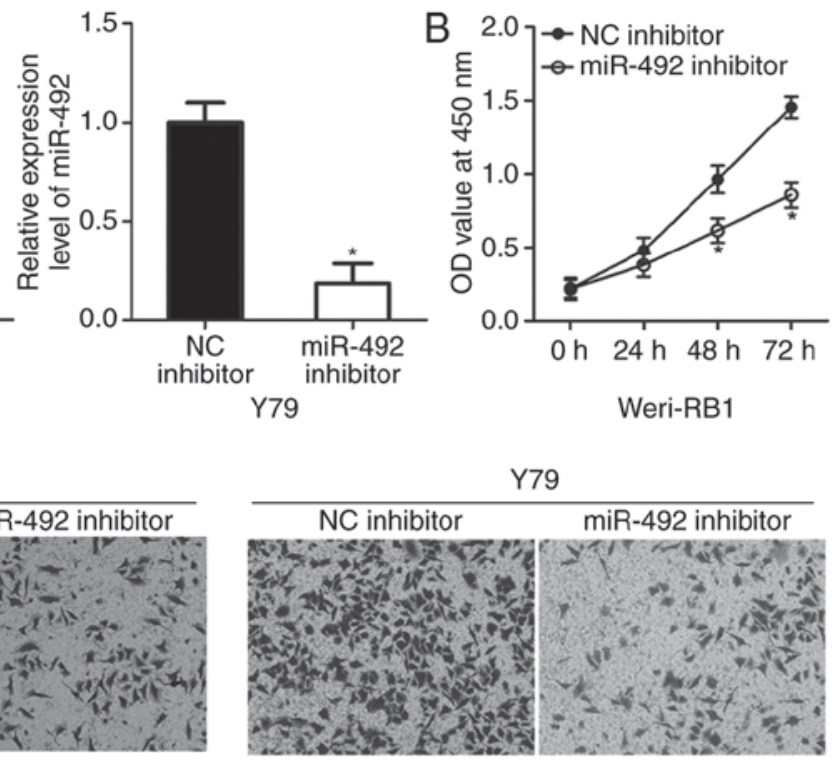
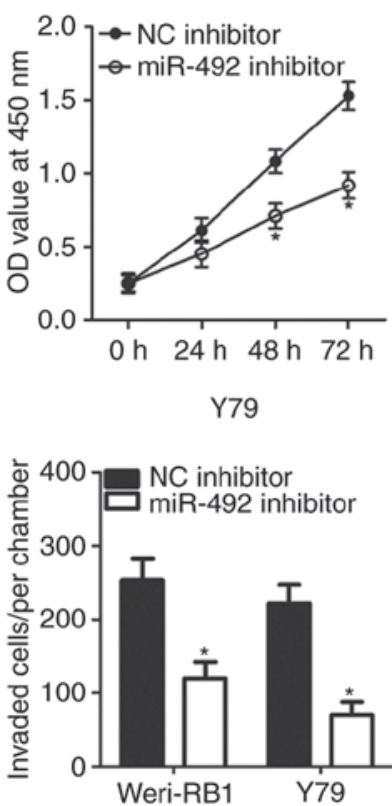

Figure 2. miR-492 inhibition attenuates proliferation and invasion of Weri-RB1 and Y79 cells. (A) Weri-RB1 and Y79 cells were treated with miR-492 inhibitor or NC inhibitor. The expression level of miR- 492 was analyzed via RT-qPCR. "P<0.05 vs. NC inhibitor. (B) CCK-8 assay was performed to evaluate the effect of miR-492 downregulation on Weri-RB1 and $\mathrm{Y} 79$ cell proliferation. " $\mathrm{P}<0.05$ vs. NC inhibitor. (C) Representative images from the invasion assay with NC inhibitor- or miR-492 inhibitor-transfected Weri-RB1 and Y79 cells. Downregulation of miR-492 inhibited the invasive abilities of Weri-RB1 and Y79 cells. ${ }^{*} \mathrm{P}<0.05$ vs. NC inhibitor.

A

LATS2-Wt-3'-UTR $\quad 5^{\prime}$...UCUUGCCAUUCACAGCAGGUCCU.... $3^{\prime}$ hsa-miR-492 $\quad 3^{\prime}$ UUCUUAGAACAGGGCGUCCAGGA $5^{\prime}$ LATS2-Mut-3'-UTR $5^{\prime}$...UCUUGCCAUUCACAGGUCCAGGU.... $3^{\prime}$
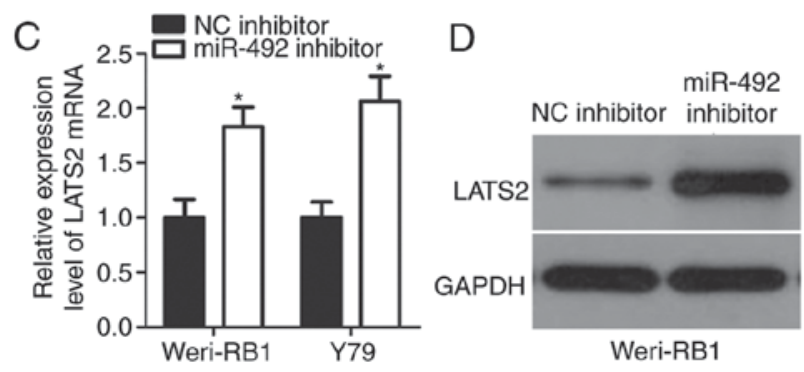
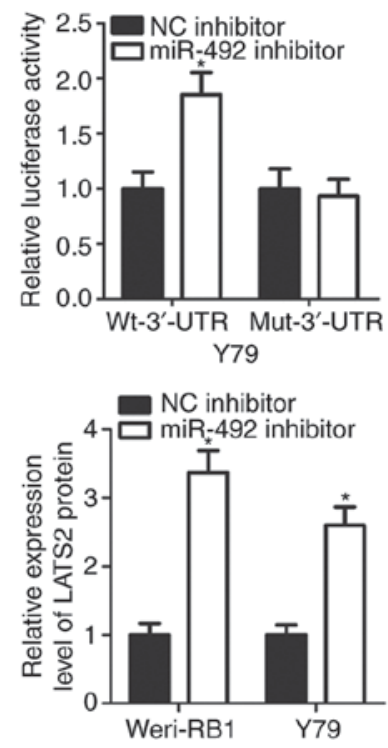

Figure 3. miR-492 directly targets LATS2 in RB cells. (A) Schematic representation of the miR-492 binding site with the 3'-UTR of wild-type LATS2 and the 3'-UTR of mutant LATS2. (B) Weri-RB1 and Y79 cells were transfected with pmirGLO-LATS2-Wt-3'-UTR or pmirGLO-LATS2-Mut-3'-UTR along with miR-492 inhibitor or NC inhibitor. The relative luciferase activities were detected at $48 \mathrm{~h}$ post-transfection using a Dual-Luciferase Reporter Assay system. "P<0.05 vs. NC inhibitor. LATS2 expression in Weri-RB1 and Y79 cells following transfection with miR-492 inhibitor or NC inhibitor was determined at the mRNA level by (C) RT-qPCR and the protein level by (D) western blot analysis. "P<0.05 vs. NC inhibitor.

as the seed sequence of miR-492 was complementary to the 3'-UTR of LATS2 at positions 705-711 (Fig. 3A). To determine whether LATS2 is a direct target of miR-492 in RB, luciferase reporter plasmids containing either the wild-type or mutant 3'-UTR of LATS2, together with miR-492 or NC inhibitor, were transfected into Weri-RB1 and Y79 cells. Co-transfection of pmirGLO-LATS2-Wt-3'-UTR and miR-492 inhibitor into Weri-RB1 and Y79 cells resulted in significantly increased luciferase activities $(\mathrm{P}<0.05)$. However, miR-492 inhibitor had none effect on the luciferase activities of the plasmid carrying the mutant binding site in the 3'-UTR of LATS2 (Fig. 3B). In addition, the present study investigated whether miR-492 can regulate endogenous LATS2 expression in Weri-RB1 and Y79 cells. Compared with the NC inhibitor group, miR-492-knockdown markedly increased the LATS2 mRNA (Fig. 3C; P<0.05) and protein (Fig. 3D; $\mathrm{P}<0.05$ ) expression levels in Weri-RB1 and Y79 cells. These findings confirmed that LATS2 is a direct target of miR-492 in RB cells. 

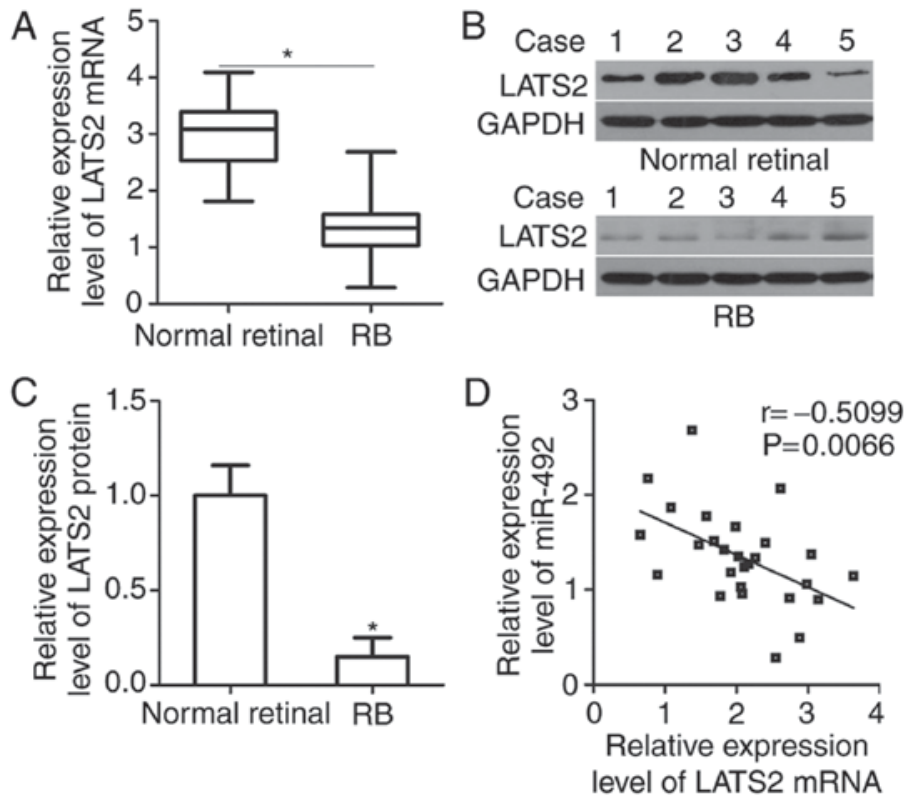

Figure 4. LATS2 is weakly expressed in RB tissues and negatively correlated with miR-492 expression. The mRNA and protein levels of LATS2 were analysed in RB tissues and normal retinal tissues through (A) RT-qPCR and (B) western blot analysis, respectively. (C) Statistical analysis of western blotting. ${ }^{*} \mathrm{P}<0.05$ vs. normal retinal tissues. (D) The association between miR-492 and LATS2 mRNA levels in RB tissues was examined using Spearman's correlation analysis. $\mathrm{r}=-0.5099, \mathrm{P}=0.0066$.

LATS2 underexpression in RB tissues is negatively correlated with miR-492 expression. To elucidate the association between miR-492 and LATS2 in RB, the expression of LATS2 in RB tissues and normal retinal tissues was detected. The results of RT-qPCR and western blot analysis revealed that LATS2 expression was downregulated in RB tissues at mRNA (Fig. 4A; $\mathrm{P}<0.05$ ) and protein (Fig. $4 \mathrm{~B}$ and $\mathrm{C} ; \mathrm{P}<0.05$ ) levels, compared with that in normal retinal tissues. Spearman's correlation analysis was adopted to illustrate the association between miR-492 and LATS2 mRNA levels in RB tissues. As demonstrated in Fig. 4D, the miR-492 and LATS2 mRNA expression levels were negatively correlated in RB tissues (Fig. 4D; $r=-0.5099, \mathrm{P}=0.0066$ ). These results suggested that the weak LATS2 expression in RB tissues is partly due to miR-492 upregulation.

LATS2 downregulation abrogates the effect of the miR-492 inhibitor in $R B$ cells. Rescue experiments were performed to investigate whether the roles of miR-492 inhibition in $\mathrm{RB}$ cells are mediated by LATS2 upregulation. LATS2-siRNA or NC-siRNA was transfected into Weri-RB1 and Y79 cells. Next, western blot analysis was used to detect LATS2 protein expression. As demonstrated in Fig. 5A, LATS2 expression was efficiently knocked down in LATS2-siRNA-transfected Weri-RB1 and Y79 cells, compared with that in cells transfected with NC-siRNA $(\mathrm{P}<0.05)$. Subsequently, LATS2-siRNA or NC-siRNA was co-transfected with miR-492 inhibitor into Weri-RB1 and Y79 cells. The results indicated that the co-transfection of LATS2-siRNA partially abolished the miR-492 inhibitor-mediated upregulation of LATS2 in Weri-RB1 and Y79 cells (Fig. 5B and C; $\mathrm{P}<0.05$ ). CCK-8 and invasion assays demonstrated that LATS2 downregulation can reverse the suppression of proliferation (Fig. 5D; P<0.05) and invasion (Fig. 5E; P<0.05) induced by the miR-492 inhibitor in Weri-RB1 and Y79 cells. Taken together, these results indicated that the oncogenic roles of miR-492 in RB cells are partly mediated by LATS2.

\section{Discussion}

Numerous studies have demonstrated that miRNAs are upregulated or downregulated in $\mathrm{RB}$, and this phenomenon is associated with the modulation of various malignant behaviours during RB occurrence and development (16-18). Therefore, the mechanisms that associate deregulated miRNAs with RB initiation and progression must be understood to identify effective therapeutic techniques for patients with RB. The present study detected miR-492 expression and investigated its biological roles in RB development. It was revealed that miR-492 expression was markedly overexpressed in RB tissues and cell lines, compared with that in normal retinal tissues and the normal retinal pigmented epithelial cell line ARPE-19, respectively. Functional experiments revealed that miR-492 downregulation impeded the proliferation and invasion of RB cells. In addition, LATS2 was identified as a direct target gene of miR-492 in RB cells, and a negative association was observed between the miR-492 and LATS 2 mRNA levels in RB tissues. LATS2 downregulation reversed the suppression of RB cell proliferation and invasion caused by miR-492 inhibition. Therefore, miR-492 inhibition may be a valuable treatment option for patients with RB.

miR-492 is aberrantly expressed in several types of human cancer.Forexample, miR-492 is downregulated incervicalcancer tissue specimens and cell lines (20). miR-492 downregulation is significantly correlated with the pelvic lymph node metastasis of patients with cervical cancer (20). Weakly expressed miR-492 has also been observed in osteosarcoma (21) and clear cell renal cell carcinoma (22). By contrast, miR-492 is highly expressed in hepatoblastoma tissues and cell lines. Retinal cells in patients with hepatoblastoma who present with high miR-492 levels exhibit more high-risk or aggressive behaviours than those with low miR-492 levels $(26,27)$. miR-492 is also upregulated in hepatic cancer, and this upregulation is strongly associated with poor survival (23). The results of these conflicting studies 
A

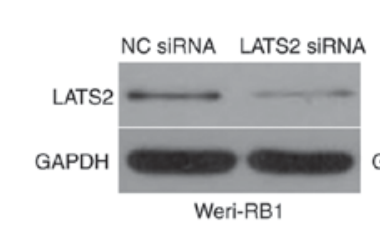

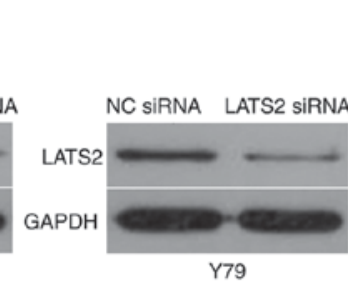


Figure 5. LATS2-knockdown mediates the effects of miR-492 inhibition on RB cells. (A) LATS2 siRNA or NC siRNA was introduced into Weri-RB1 and Y79 cells. Western blot analysis was used to evaluate the transfection efficiency. $\mathrm{P}<0.05$ vs. NC siRNA. Weri-RB1 and Y79 cells were transfected with (B) LATS2-siRNA or NC-siRNA, and (C) with miR-492 inhibitor. LATS2 protein expression was measured by western blot analysis. ${ }^{\text {P }<0.05}$ vs. NC inhibitor. ${ }^{\#} \mathrm{P}<0.05$ vs. miR-492 inhibitor + LATS2-siRNA. (D) CCK-8 and (E) invasion assays were conducted to determine proliferative and invasion abilities of indicated cells. ${ }^{*} \mathrm{P}<0.05$ vs. NC inhibitor. ${ }^{\#} \mathrm{P}<0.05$ vs. miR-492 inhibitor + LATS2-siRNA.

suggested that the expression pattern of miR-492 exhibits tissue specificity, making it a promising biomarker for diagnosing different types of human cancer.

miR-492 serves as a tumour suppressor in numerous types of human cancer. miR-492 upregulation inhibits the proliferation and invasion of cervical cancer cells, and increases the sensitivity of cells to irradiation by inducing apoptosis (20). In osteosarcoma, the resumption of miR-492 expression suppresses cell growth and metastasis in vitro, and decreases in vivo tumour growth (21). In clear cell renal cell carcinoma, miR-492 restoration restricts cell proliferation and invasion, induces cell apoptosis and promotes cell adhesion (22). Nonetheless, miR-492 serves oncogenic roles in hepatoblastoma by promoting cell proliferation, anchorage-independent growth and metastasis (26). In breast cancer, ectopic miR-492 expression significantly promotes cell proliferation and anchorage-independent growth (24). In hepatic cancer, miR-492 downregulation attenuates cell proliferation in vitro and restricts tumour growth in vivo (23). These conflicting findings indicated that the biological roles of miR-492 exhibit tissue specificity. Considering its crucial roles in tumorigenesis and tumor development, a miR-492-based targeted therapy may be effective for the treatment of patients with these caner types.

Researchers have identified numerous target genes of miR-492, including TIMP2 (20) in cervical cancer, PAK7 (21) in osteosarcoma, CD44 (26) in hepatoblastoma, SOX7 (24) in breast cancer and PTEN (23) in hepatic cancer. LATS2, which is a member of the serine/threonine AGC kinase family, was validated as a direct target of miR-492 in RB. It is located at chromosome $13 \mathrm{q} 11-12$ and is highly conserved from flies to humans (28). Decreased LATS2 expression has been reported in various cancer types, including acute lymphoblastic leukaemia (29), breast cancer (30) and prostate cancer (31). LATS2 is downregulated in RB and is associated with the formation and progression of RB (32). The present study demonstrated that miR-492 inhibition directly targeted LATS2 to inhibit the development of RB. Overall, these results confirmed that a miR-492/LATS2-based targeted therapy may be a novel and efficient therapeutic strategy for patients with RB.

In conclusion, the results of the present study revealed that miR-492 was upregulated in RB tissues and cell lines, and its downregulation significantly inhibited the proliferation and invasion of RB cells. LATS2 was a direct target gene of miR-492 in RB. Considering these results, we hypothesized that miR-492-knockdown or the restoration of LAST2 expression may be an attractive therapeutic target for patients with RB. However, the present study did not analyse the effects of miR-492 underexpression on RB cells in vivo. This is a limitation of the present study and will be the focus of our future experiments. 


\section{Acknowledgements}

Not applicable.

\section{Funding}

No funding was recieved.

\section{Availability of data and materials}

The datasets used and/or analyzed during the present study are available from the corresponding author on reasonable request.

\section{Authors' contributions}

AZ designed the present study. ZS, AZ and LZ performed the functional experiments. All authors have read and approved the final draft.

\section{Ethics approval and consent to participate}

The present study was approved by the Ethics Committee of Weifang People's Hospital and was performed in accordance with the Declaration of Helsinki and the guidelines of the Ethics Committee of Weifang People's Hospital. Written informed consent was obtained from all patients for the use of their clinical tissues.

\section{Patients consent for publication}

Not applicable.

\section{Competing interests}

The authors declare that they have no competing interests.

\section{References}

1. Dimaras H, Corson TW, Cobrinik D, White A, Zhao J, Munier FL, Abramson DH, Shields CL, Chantada GL, Njuguna F and Gallie BL: Retinoblastoma. Nat Rev Dis Primers 1: 15021, 2015.

2. Broaddus E, Topham A and Singh AD: Incidence of retinoblastoma in the USA: 1975-2004. Br J Ophthalmol 93: 21-23, 2009.

3. MacCarthy A, Draper GJ, Steliarova-Foucher E and Kingston JE: Retinoblastoma incidence and survival in European children (1978-1997). Report from the Automated Childhood Cancer Information System project. Eur J Cancer 42: 2092-2102, 2006.

4. Benavente CA and Dyer MA: Genetics and epigenetics of human retinoblastoma. Annu Rev Pathol 10: 547-562, 2015.

5. Shields CL and Shields JA: Retinoblastoma management: Advances in enucleation, intravenous chemoreduction, and intra-arterial chemotherapy. Curr Opin Ophthalmol 21: 203-212, 2010.

6. Dimaras H, Dimba EA and Gallie BL: Challenging the global retinoblastoma survival disparity through a collaborative research effort. Br J Ophthalmol 94: 1415-1416, 2010.

7. Wu X, Zeng Y, Wu S, Zhong J, Wang Y and Xu J: MiR-204, down-regulated in retinoblastoma, regulates proliferation and invasion of human retinoblastoma cells by targeting CyclinD2 and MMP-9. FEBS Lett 589: 645-650, 2015.

8. Bartel DP: MicroRNAs: Genomics, biogenesis, mechanism, and function. Cell 116: 281-297, 2004.

9. Cai Y, Yu X, Hu S and Yu J: A brief review on the mechanisms of miRNA regulation. Genomics Proteomics Bioinformatics 7: $147-154,2009$

10. Pratap P, Raza ST, Abbas S and Mahdi F: MicroRNA-associated carcinogenesis in lung carcinoma. J Cancer Res Ther 14: 249-254, 2018.
11. Vannini I, Fanini F and Fabbri M: Emerging roles of microRNAs in cancer. Curr Opin Genet Dev 48: 128-133, 2018.

12. Lou W, Liu J, Gao Y, Zhong G, Chen D, Shen J, Bao C, Xu L, Pan J, Cheng J, et al: MicroRNAs in cancer metastasis and angiogenesis. Oncotarget 8: 115787-115802, 2017.

13. Liu S, Zhang X, Hu C, Wang Y and Xu C: miR-29a inhibits human retinoblastoma progression by targeting STAT3. Oncol Rep 39: 739-746, 2018.

14. Wang Z, Yao YJ, Zheng F, Guan Z, Zhang L, Dong N and Qin WJ: Mir-138-5p acts as a tumor suppressor by targeting pyruvate dehydrogenase kinase 1 in human retinoblastoma. Eur Rev Med Pharmacol Sci 21: 5624-5629, 2017.

15. Yang L, Wei N, Wang L, Wang X and Liu QH: miR-498 promotes cell proliferation and inhibits cell apoptosis in retinoblastoma by directly targeting CCPG1. Childs Nerv Syst 34: 417-422, 2018.

16. Zhang M, Li Q, Pan Y, Wang H, Liu G and Yin H: MicroRNA-655 attenuates the malignant biological behaviours of retinoblastoma cells by directly targeting PAX6 and suppressing the ERK and p38 MAPK signalling pathways. Oncol Rep 39: 2040-2050, 2018.

17. Singh U, Malik MA, Goswami S, Shukla S and Kaur J: Epigenetic regulation of human retinoblastoma. Tumour Biol 37: 14427-14441, 2016.

18. Golabchi K, Soleimani-Jelodar R, Aghadoost N, Momeni F, Moridikia A, Nahand JS, Masoudifar A, Razmjoo H and Mirzaei H: MicroRNAs in retinoblastoma: Potential diagnostic and therapeutic biomarkers. J Cell Physiol 233: 3016-3023, 2018.

19. Xu X, Ge S, Jia R, Zhou Y, Song X, Zhang H and Fan X: Hypoxia-induced miR-181b enhances angiogenesis of retinoblastoma cells by targeting PDCD10 and GATA6. Oncol Rep 33: 2789-2796, 2015 .

20. Liu M, An J, Huang M, Wang L, Tu B, Song Y, Ma K, Wang Y, Wang S, Zhu H, et al: MicroRNA-492 overexpression involves in cell proliferation, migration, and radiotherapy response of cervical squamous cell carcinomas. Mol Carcinog 57: 32-43, 2018.

21. Song X, Xie Y, Liu Y, Shao M and Yang W: MicroRNA-492 overexpression exerts suppressive effects on the progression of osteosarcoma by targeting PAK7. Int J Mol Med 40: 891-897, 2017.

22. Wu A, Wu K, Li M, Bao L, Shen X, Li S, Li J and Yang Z: Upregulation of microRNA-492 induced by epigenetic drug treatment inhibits the malignant phenotype of clear cell renal cell carcinoma in vitro. Mol Med Rep 12: 1413-1420, 2015.

23. Jiang J, Zhang Y, Yu C, Li Z, Pan Y and Sun C: MicroRNA-492 expression promotes the progression of hepatic cancer by targeting PTEN. Cancer Cell Int 14: 95, 2014

24. Shen F, Cai WS, Feng Z, Li JL, Chen JW, Cao J and Xu B: MiR-492 contributes to cell proliferation and cell cycle of human breast cancer cells by suppressing SOX7 expression. Tumour Biol 36: 1913-1921, 2015.

25. Livak KJ and Schmittgen TD: Analysis of relative gene expression data using real-time quantitative PCR and the 2(-Delta Delta C(T)) method. Methods 25: 402-408, 2001.

26. von Frowein J, Hauck SM, Kappler R, Pagel P, Fleischmann KK, Magg T, Cairo S, Roscher A, von Schweinitz D and Schmid I: MiR-492 regulates metastatic properties of hepatoblastoma via CD44. Liver Int 38: 1280-1291, 2018.

27. von Frowein J, Pagel P, Kappler R, von Schweinitz D, Roscher A and Schmid I: MicroRNA-492 is processed from the keratin 19 gene and up-regulated in metastatic hepatoblastoma. Hepatology 53: 833-842, 2011.

28. Pearce LR, Komander D and Alessi DR: The nuts and bolts of AGC protein kinases. Nat Rev Mol Cell Biol 11: 9-22, 2010.

29. Jimenez-Velasco A, Roman-Gomez J, Agirre X, Barrios M, Navarro G, Vazquez I, Prosper F, Torres A and Heiniger A: Downregulation of the large tumor suppressor 2 (LATS2/KPM) gene is associated with poor prognosis in acute lymphoblastic leukemia. Leukemia 19: 2347-2350, 2005.

30. Takahashi Y, Miyoshi Y, Morimoto K, Taguchi T, Tamaki Y and Noguchi S: Low LATS2 mRNA level can predict favorable response to epirubicin plus cyclophosphamide, but not to docetaxel, in breast cancers. J Cancer Res Clin Oncol 133: 501-509, 2007.

31. Powzaniuk M, McElwee-Witmer S, Vogel RL, Hayami T, Rutledge SJ, Chen F, Harada S, Schmidt A, Rodan GA, Freedman LP and Bai C: The LATS2/KPM tumor suppressor is a negative regulator of the androgen receptor. Mol Endocrinol 18: 2011-2023, 2004

32. Chakraborty S, Khare S, Dorairaj SK, Prabhakaran VC, Prakash DR and Kumar A: Identification of genes associated with tumorigenesis of retinoblastoma by microarray analysis. Genomics 90: 344-353, 2007. 